\title{
Referential Opacity In Nondeterministic Data Refinement
}

\author{
XIAOLEI QIAN and ALLEN GOLDBERG \\ Kestrel Institute
}

\begin{abstract}
Data refinement is the transformation in a program of one data type to another. With the obvious formalization of nondeterministic data types in equational logic however, many desirable nondeterministic data refinements are impossible to prove correct. Furthermore, it is difficult to have a monotonic notion of refinement. We propose an alternative formalization of nondeterministic data types, in which the requirement of referential transparency applies only to deterministic operators. We show how the above-mentioned problems can be solved with our approach.

Categories and Subject Descriptors: D.2.4 [Software Engineering]: Program Verification-correctness proofs; D.3.3 [Programming Languages]: Language Constructs and Features-abstract data types; F.3.2 [Logics and Meanings of Programs]: Semantics of Programming Languages-algebraic approaches to semantics
\end{abstract}

General Terms: Languages, Theory, Verification

Additional Key Words and Phrases: Algebraic specification, data refinement, nondeterminism, program transformation, referential transparency, theory morphism

\section{INTRODUCTION}

Data refinement is the transformation in a program of one data type to another. Data refinement has been approached by formalizing the semantics of abstract data types by initial algebras [Guttag and Horning 1978], data type specifications by algebraic theories in equational logic [Bidoit et al. 1991], and (correct) data refinement by theory morphisms [Goguen et al. 1987; Sannella and Tarleck; 1988]. Such formalization has the nice property

This work was supported by the U.S. Air Force Rome Laboratory under contract F30602-86-C0026 .

Authors' addresses: X. Qian, Computer Science Laboratory, SRI International, 333 Ravenswood Avenue, Menlo Park, CA 94025; A. Goldberg, Kestrel Institute, 3260 Hillview Avenue, Palo Alto, CA 94304.

Permission to copy without fee all or part of this material is granted provided that the copies are not made or distributed for direct commercial advantage, the ACM copyright notice and the title of the publication and its date appear, and notice is given that copying is by permission of the Association for Computing Machinery. To copy otherwise, or to republish, requires a fee and/or specific permission.

(C) 1993 ACM 1057-4514/93/0300-0233\$03.50

ACM Letters on Programming Languages and Systems,

Vol. 2, Nos 1-4, March-December 1993, Pages 233-241. 
that, assuming abstract data type $A$ is refined to abstract data type $B$, replacing $A$ by $B$ in program $P$ preserves the correctness of $P$ [Broy et al. 1986].

Nondeterminism provides a convenient vehicle to avoid specifying all details of an implementation prematurely. The stepwise refinement of a specification to an implementation can be viewed as a process in which nondeterminism is gradually removed by making design decisions [Turski and Maibaum 1987]. The semantics of nondeterministic data types has been formalized as multialgebras [Hesselink 1988; Nipkow 1986], which essentially avoids nondeterminism by encapsulating it through the medium of set construction. However, the straightforward formulation of nondeterministic data type specifications as algebraic theories in equational logic makes many desirable data refinements impossible to prove correct, due to the requirement of referential transparency.

Consider for example the refinement of SET to SEQUENCE [Blaine and Goldberg 1991]. It is desirable to refine equality of SET not to equality of SEQUENCE but to an equivalence relation of SEQUENCE, in which sequences that are permutations of the same set are equivalent. Meanwhile, it is also desirable to refine the nondeterministic choose operator of SET, which chooses an element from a nonempty set, to the head operator of SEQUENCE, which takes the first element of a nonempty sequence. Obviously these two data refinements cannot coexist in the presence of referential transparency: $x=y$ $\rightarrow \operatorname{choose}(x)=\operatorname{choose}(y)$.

Besides formalizing data refinements as theory morphisms, data refinements can also be specified as conditional refinement rules of the form $\phi: t \Longrightarrow t^{\prime}$, which are formalized as conditional equations in data type specifications. However, many desirable refinement rules for nondeterministic data types lead to inconsistent specifications under this formalization. For example, we would like to have a conditional refinement rule in our specification of SET: $x \neq\{\}: \operatorname{choose}(\mathrm{x} \cup \mathrm{y}) \Rightarrow \operatorname{choose}(x)$, which is formalized as a conditional equation in the algebraic theory of set: $x \neq\{\} \rightarrow \operatorname{choose}(x \cup y)=\operatorname{choose}(x)$. Assuming $a \neq b$, we would have choose $(\{a\} \cup\{b\}) \Longrightarrow \operatorname{choose}(\{a\})$ and choose $(\{b\} \cup\{a\}) \Rightarrow$ choose $(\{b\})$. Because of referential transparency, they lead to $a=b$, which is clearly inconsistent.

Referential transparency and related issues were studied in detail in Søndergaard and Sestoft [1990]. It was recognized in Bird et al. [1987] that one might have to give up referential transparency in order to adequately deal with nondeterminism. There it was suggested that a well-defined notion of refinement should be reflexive, transitive, and monotonic. Meseguer observed in 1992 that term rewriting should not be formalized in equational logic for applications such as nondeterministic data types, concurrent systems, and object-oriented computation.

We propose an alternative formalization of nondeterministic data types in which referential transparency applies only to deterministic operators (Section 2). Based on this formalization, we show how various desirable data refinements can be proven correct (Section 3), and how a well-defined notion 
of refinement can be incorporated into the formalism (Section 4). Finally we conclude the paper in Section 5.

\section{NONDETERMINISTIC DATA TYPES}

A signature $\sum$ is a pair $(S, \Omega)$, where $S$ is a set of sort symbols and $\Omega$ is a family of finite disjoint sets $\left\{\Omega_{v, s}\right\}_{v \in S^{*}, s \in S}$ of operator symbols. $\Omega$ is divided into two disjoint families $\Omega^{d}$ and $\Omega^{n}$. Operator symbols in $\Omega^{d}$ are deterministic, while operator symbols in $\Omega^{n}$ are nondeterministic. We write $f: v \rightarrow s$ to denote $v \in S^{*}, s \in S$, and $f \in \Omega_{v, s}$. Let bool be the sort symbol for truth values, $\Omega_{v, b o o l}$ is a set of predicate symbols for $v \in S^{*}$. For every sort $s \in S$ we assume that there is an (infix) predicate symbol $=_{s} \in \Omega_{s, s, b o o l}$. The logical connectives $\vee, \wedge, \neg, \rightarrow, \leftrightarrow$ are treated as boolean operator symbols.

For signature $\sum=(S, \Omega)$, the $\sum$-terms are defined inductively as the wellsorted composition of sorted variables and operator symbols in $\Omega$. A $\sum$-term $t$ is deterministic if all operator symbols in $t$ are from $\Omega^{d}$. Otherwise $t$ is nondeterministic. A $\sum$-formula is a formula built from $\sum$-terms and quantifiers $\forall$ and $\exists$. A $\sum$-sentence is a closed $\sum$-formula.

Let $\sum=\langle S, \Omega\rangle$ be a signature. A (partial) $\sum$-algebra $\mathscr{A}$ consists of an $S$-indexed family of carrier sets $A=\left\{A_{s}\right\}_{s \in S}$; a (partial) function $f_{A}: A_{v_{1}}$ $\times \cdots \times A_{v_{n}} \rightarrow A_{s}$ for every $f \in \Omega_{v, s}^{d}$, where $v=\left\langle v_{1}, \ldots, v_{n}\right\rangle$; and a (partial) function $f_{A}: A_{v_{1}} \times \cdots \times A_{v_{n}} \rightarrow P\left(A_{s}\right)$ for every $f \in \Omega_{v, s}^{n}$, where $v=$ $\left\langle v_{1}, \ldots, v_{n}\right\rangle$ and $P\left(A_{s}\right)$ denotes the set of nonempty subsets of $A_{s}$.

A nondeterministic data type $T$ is a pair $\langle\Sigma, \Phi\rangle$, where $\Sigma=\langle S, \Omega\rangle$ is a signature and $\Phi$ is a set of $\sum$-sentences called axioms. For every sort $s \in S$ we assume that the following equality axioms are in $\Phi$ :

(1) Reflexivity $(\forall x)\left(x={ }_{s} x\right)$,

(2) Symmetry $(\forall x, y)\left(x=_{s} y \rightarrow y=_{s} x\right)$,

(3) Transitivity $(\forall x, y, z)\left(x=y \wedge y={ }_{s} z \rightarrow x={ }_{s} z\right)$,

(4) Substitutivity For $f \in \Omega_{v, s}^{d}$ where $v=\left\langle v_{1}, \ldots, v_{l}, \ldots, v_{n}\right\rangle$,

$$
\begin{aligned}
& \left(\forall x, y, z_{1}, \ldots, z_{i-1}, z_{\imath+1}, \ldots, z_{n}\right) \\
& \left(x={ }_{v_{\iota}} y \rightarrow f\left(z_{1}, \ldots, z_{\iota-1}, x, z_{\iota+1}, \ldots, z_{n}\right)\right. \\
& \left.\quad{ }_{s} f\left(z_{1}, \ldots, z_{i-1}, y, z_{\imath+1}, \ldots, z_{n}\right)\right)
\end{aligned}
$$

The substitutivity axiom schema applies only to deterministic operators, meaning that we do not enforce referential transparency on nondeterministic operators. A $T$-model is a $\sum$-algebra that satisfies the axioms of $T$. A $\sum$-sentence $p$ is a $T$-theorem, denoted as $T \vDash p$, if $p$ is a logical consequence of the axioms of $T$. T-theory is the set of $T$-theorems. As examples, the following are the specifications of two partial and nondeterministic data types: SET and SEQ, both of which take ATOM as a parameter data type with sort atom and operator $f\left(\_\right)$: atom $\rightarrow$ atom. 


$$
\begin{aligned}
& \operatorname{SET}(\text { ATOM }) \stackrel{\text { def }}{=} \text { (sorts set } \\
& \text { deterministic operators } \quad\{\}: \rightarrow \text { set } \\
& { }^{\circ}{ }^{\circ}: \text { atom, set } \rightarrow \text { set } \\
& \epsilon_{-}: \text {atom, set } \rightarrow \text { bool } \\
& \left.\operatorname{apply} f_{(}\right): \text {set } \rightarrow \text { set } \\
& \text { nondeterministic operators choose (_); set } \rightarrow \text { atom } \\
& \text { axioms } \quad \neg(a \circ S=\{\}) \\
& a \circ(b \circ S)=b \circ(a \circ S) \\
& a \circ(a \circ S)=a \circ S \\
& \neg(a \in\{\}) \\
& a \in(b \circ S) \leftrightarrow a=b \vee a \in S \\
& \operatorname{apply} f(\{\})=\{\} \\
& \operatorname{apply} f(a \circ S)=f(a) \circ \operatorname{apply}^{f}(S) \\
& \neg(S=\{\}) \rightarrow \operatorname{choose}(S) \in S) \\
& \text { seq } \\
& \text { [ ]: } \rightarrow \operatorname{seq} \\
& { }^{\circ}{ }_{-} \text {: atom, seq } \rightarrow \mathrm{seq} \\
& \text { head(_): seq } \rightarrow \text { atom } \\
& \epsilon_{-}: \text {atom, seq } \rightarrow \text { bool } \\
& \text { axioms } \\
& \operatorname{apply} f_{(-)}: \operatorname{seq} \rightarrow \operatorname{seq} \\
& \neg(a \circ Q=[]) \\
& a \circ Q=b \circ Q^{\prime} \rightarrow a=b \wedge Q=Q^{\prime} \\
& \operatorname{head}(a \circ Q)=a \\
& \neg(a \in[]) \\
& a \in(b \circ Q) \leftrightarrow a=b \vee a \in Q \\
& \text { apply } f([])=[] \\
& \operatorname{apply} f(a \circ Q)=f(a) \circ \operatorname{apply} f(Q))
\end{aligned}
$$

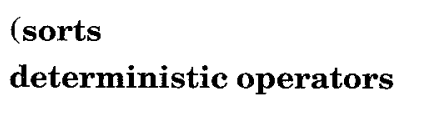

The intuition behind the nondeterministic choose operator of SET is that it can be referentially opaque. We do not expect that choose $(S)$ computes a specific element of $S$, nor that choose $(S)$ always computes the same element of $S$. All we require about choose is given by the last axiom of SET.

\section{DATA REFINEMENT}

A signature morphism $\sigma: \Sigma \rightarrow \Sigma^{\prime}$, where $\Sigma=\langle S, \Omega\rangle$ and $\Sigma^{\prime}=\left\langle S^{\prime}, \Omega^{\prime}\right\rangle$, is a pair $\langle\delta, \theta\rangle$ where $\delta: S \rightarrow S^{\prime}$ is a map and $\theta$ is a family of maps $\left\{\theta_{v, s}: \Omega_{v, s} \rightarrow\right.$ $\left.\Omega_{\delta^{*}(v), \delta(s)}^{\prime}\right\}_{v \in S^{*}, s \in S}$ where $\delta^{*}\left(\left\langle v_{1}, \ldots, v_{n}\right\rangle\right)$ denotes $\left\langle\delta\left(v_{1}\right), \ldots, \delta\left(v_{n}\right)\right\rangle$ for $v_{1}, \ldots, v_{n} \in S$. We write $\sigma(s)$ for $\delta(s), \sigma(v)$ for $\delta^{*}(v)$, and $\sigma(f)$ for $\theta_{v, s}(f)$ where $f \in \Omega_{v, s}$. Given a $\sum$-formula $p, \sigma(p)$ denotes the $\Sigma^{\prime}$-formula resulted from replacing every variable of sort $s$ in $p$ by a distinct variable of sort $\sigma(s)$, and every operator symbol $f$ in $p$ by $\sigma(f)$. An obvious signature 
morphism from SET to $\mathrm{SEQ}$ is the following:

$$
\begin{aligned}
& \{\text { set } \mapsto s e q \text {, } \\
& \{\} \mapsto[],{ }_{s e t} \mapsto \circ_{s e q}, \in_{s e t} \mapsto \in_{\text {seq }}, \text { apply } \text { set }_{\text {set }}^{f} \mapsto \text { apply }_{\text {seq }}^{f}, \\
& \left.=_{\text {set }} \mapsto=_{\text {seq }} \text {, choose } \mapsto \text { head }\right\}
\end{aligned}
$$

Let $T=\langle\Sigma, \Phi\rangle$ and $T^{\prime}=\left\langle\Sigma^{\prime}, \Phi^{\prime}\right\rangle$ be two nondeterministic data types, and $\sigma: \Sigma \rightarrow \Sigma^{\prime}$ be a signature morphism. We say that $\sigma$ is a data refinement from $T$ to $T^{\prime}$ if $T^{\prime} \vDash \sigma(A)$ for every axiom $A \in \Phi$. Obviously the above signature morphism from SET to SEQ is not a data refinement, because SEQ $¥ a \circ(a \circ Q)$ $=a \circ Q$.

Suppose that $T_{1}, T_{2}$ are two nondeterministic data types with equality predicates $={ }_{1},=_{2}$ respectively. In refining $T_{1}$ to $T_{2}$, a critical decision is how to refine equality. There are two possible ways that $=_{1}$ can be refined through signature morphism $\sigma$.

(1) If $\sigma\left(=_{1}\right)$ is $=_{2}$, then we are often forced to require more knowledge from $T_{1}$. Moreover, it might add too much detail that prohibits certain efficient implementations. For example, if $\sigma\left(=_{\text {set }}\right)$ is $=_{\text {seq }}$, then $\sigma\left({ }^{\circ}{ }_{\text {set }}\right)$ cannot be ${ }^{\circ}$ seq. We might require that there is a total ordering $\leq$ on atom, and build into SEQ an (ordered) insert operator _ $\diamond_{-}:$atom, seq $\rightarrow$ seq defined by

$$
\begin{aligned}
& a \diamond[]=a \circ[] \\
& a \diamond(a \circ Q)=a \circ Q \\
& a<b \rightarrow a \diamond(b \circ Q)=a \circ(b \circ Q) \\
& b<a \rightarrow a \diamond(b \circ Q)=b \circ(a \diamond Q)
\end{aligned}
$$

Now $\sigma\left({ }^{\circ}{ }_{\text {set }}\right)$ can be $\diamond$. This implementation represents every set as an ordered sequence with no duplicate atoms. It is less efficient when ${ }^{\circ}{ }_{\text {set }}$ is used more frequently than $=_{\text {set }}$.

(2) If $\sigma\left(=_{1}\right)$ is $\approx$ different from $=_{2}$, then $\approx$ must be logically weaker than $=_{2}$, namely for all $x, y$ we have $x={ }_{2} y$ implies $x \approx y$, because $\approx$ must satisfy all the equality axioms. The refinement of $=_{1}$ is underspecified: every object of $T_{1}$ is refined to a group of objects of $T_{2}$ not distinguishable under $\approx$. This delay of implementation decisions is essential in keeping more implementation options available. For example, we might build into $\mathrm{SEQ}$ a range containment predicate $\_\complement_{-}: s e q, s e q \rightarrow$ bool and a range equality predicate ${ }_{-}=_{r-}: s e q, s e q \rightarrow$ bool defined by:

$$
\begin{aligned}
& \text { [ ] } 5 Q \\
& a \circ Q \sqsubseteq Q^{\prime} \leftrightarrow a \in Q^{\prime} \wedge Q \sqsubseteq Q^{\prime} \\
& Q={ }_{r} Q^{\prime} \leftrightarrow Q \sqsubseteq Q^{\prime} \wedge Q^{\prime} \sqsubseteq Q
\end{aligned}
$$

Now $\sigma\left(=_{s e t}\right)$ can be $={ }_{r}$, and $\sigma\left(\circ_{s e t}\right)$ can be ${ }_{s e q}$. This implementation represents every set by a group of sequences not distinguishable under 
${ }_{r}$ : these sequences all contain the same set of atoms. It is more efficient when ${ }^{\circ}{ }_{\text {set }}$ is used more frequently than ${ }{ }_{\text {set }}$.

Suppose that $T_{1}$ has a nondeterministic operator $f: v \rightarrow s$. Another critical decision is how to refine $f$ such that the right amount of nondeterminism is removed from $T_{1}$.

(1) We might refine $f$ to a nondeterministic operator. For example, we might build into SEQ a nondeterministic operator choose seq $_{-}($) : seq $\rightarrow$ atom defined by

$$
\neg(Q=[]) \rightarrow \operatorname{choose}(Q) \in Q
$$

and refine choose $_{\text {set }}$ to it. Since data refinement can be viewed as the gradual removal of nondeterminism, this refinement causes unnecessary delay of implementation decisions.

(2) We might refine $f$ to a deterministic operator. For example, if there is a min operator on atom, we might build into SEQ a select (minimum) operator select(__): seq $\rightarrow$ atom defined by

$$
\begin{aligned}
& \operatorname{select}(a \circ[])=a \\
& \operatorname{select}(a \circ(b \circ Q))=\min (a, \operatorname{select}(b \circ Q))
\end{aligned}
$$

and refine choose $_{\text {set }}$ to it. This implementation represents arbitrary selection in a set by the selection of the minimal element of a sequence, which is linear in the size of the sequence. It destroys the flexibility about choose in SET by requiring that the implementation of choose $(S) \mathrm{com}$ putes a definite and specific element of $S$. Alternatively, we might refine choose to head, which is a constant-time operation that preserves the flexibility about choose in SET.

If referential transparency is enforced on nondeterministic operators however, certain desirable refinement combinations of equality and nondeterministic operators become incorrect. In general, if $\sigma\left(=_{v}\right)$ is $\approx, \approx$ is weaker than $=_{\sigma(v)}, f: v \rightarrow s$, and $\sigma(f)$ is deterministic, the $\sigma(f)$ has to satisfy the additional requirement that $x \approx y \rightarrow \sigma(f)(x)=_{\sigma(s)} \sigma(f)(y)$, or else the refinement is incorrect. For example, with referential transparency imposed on choose $_{\text {set }}$, the following signature morphism from SET to SEQ is not a data refinement, since $\mathrm{SEQ} \not \neq Q={ }_{r} Q^{\prime} \rightarrow \operatorname{head}(Q)=\operatorname{head}\left(Q^{\prime}\right)$.

$$
\begin{aligned}
& \{\text { set } \\
& \quad\{\text { seq }, \\
& \quad\{\} \mapsto[], o_{\text {set }} \mapsto \circ_{\text {seq }}, \in_{\text {set }} \mapsto \in_{\text {seq }}, \text { apply } y_{\text {set }}^{f} \mapsto a p p l y_{\text {seq }}^{f},=_{\text {set }} \mapsto=_{r}, \\
& \quad \text { choose } \mapsto \text { head }\}
\end{aligned}
$$

Under our approach where nondeterministic operators are referentially opaque, it is easy to verify that the above signature morphism is indeed a data refinement. It does not require additional knowledge on atom such as a total ordering or a min operator, and it provides constant-time implementation for both ${ }^{\circ}{ }_{\text {set }}$ and choose ${ }_{\text {set }}$. The combination of ${ }_{r}$ and head operators ACM Letters on Programming Languages and Systems, Vol. 2, Nos. 1-4, March-December 1993. 
in SEQ captures the essence of nondeterministic behavior exhibited by $=$ and choose operators in SET.

\section{REFINEMENT PREDICATE}

To capture the notion of refinement rules in nondeterministic data type refinement, we introduce a refinement predicate. Given a nondeterministic data type $T=\langle\Sigma, \Phi\rangle$ where $\Sigma=\langle S, \Omega\rangle$, we extend $T$ with a refinement predicate $\Rightarrow{ }_{s} \in \Omega_{s, s, b o o l}$ for $s \in S$. For every sort $s \in S$ we assume that the following refinement axioms are in $\Phi$ :

(1) Reflexivity $(\forall x)\left(x \Rightarrow{ }_{s} x\right)$;

(2) Transitivity $(\forall x, y, z)\left(x \Rightarrow{ }_{s} y \wedge y \Rightarrow{ }_{s} z \rightarrow x \Rightarrow{ }_{s} z\right)$;

(3) Substitutivity For $f \in \mathbf{\Omega}_{v, s}$ where $v=\left\langle v_{1}, \ldots, v_{l}, \ldots, v_{n}\right\rangle$,

$$
\begin{aligned}
& \left(\forall x, y, z_{1}, \ldots, z_{\imath-1}, z_{\imath+1}, \ldots, z_{n}\right) \\
& \quad\left(x \Rightarrow_{v_{\imath}} y \rightarrow f\left(z_{1}, \ldots, z_{\imath-1}, x, z_{i+1}, \ldots, z_{n}\right)\right. \\
& \left.\quad \Rightarrow{ }_{s} f\left(z_{1}, \ldots, z_{i-1}, y, z_{\imath+1}, \ldots, z_{n}\right)\right)
\end{aligned}
$$

(4) Equality $t \Rightarrow{ }_{s} t^{\prime} \rightarrow t={ }_{s} t^{\prime}$.

Compared with the equality axioms of Section 2, the substitutivity axiom schema applies to nondeterministic as well as deterministic operators. The last axiom says that the refinement predicate is stronger than the equality predicate: there are $\sum$-terms $t, t^{\prime}$ such that $t={ }_{s} t^{\prime}$ without $t \Rightarrow_{s} t^{\prime}$.

A conditional refinement rule $\phi: t \Longrightarrow t^{\prime}$ in the refinement of nondeterministic data type $T=\left\langle\sum, \Phi\right\rangle$, where $\phi$ is a $\sum$-formula and $t, t^{\prime}$ are $\sum$-terms, is expressed by a (universally quantified) $\sum$-sentence $p$ of the form $\phi \rightarrow t \Rightarrow t^{\prime}$. The application of this rule to $T$ is a nondeterministic data type $T^{\prime}=\left\langle\Sigma, \Phi^{\prime}\right\rangle$ where $\Phi^{\prime}=\Phi \cup\{p\}$. Obviously $T^{\prime}$ is a data refinement of $T$ because identity mapping is a signature morphism from $T$ to $T^{\prime}$ that preserves all axioms. Conditional refinement rules can be used both to reduce nondeterminism and to obtain optimal implementation of nondeterministic data types. As an example, we can refine SET by the following conditional refinement rules:

$$
\begin{aligned}
& \neg(S=\{\}): \operatorname{choose}\left(S \cup S^{\prime}\right) \Longrightarrow \operatorname{choose}(S) \\
& \neg(S=\{\}): \operatorname{choose}(\operatorname{apply} f(S)) \Rightarrow(\operatorname{choose}(S))
\end{aligned}
$$

The resulting nondeterministic data type $\mathrm{SET}^{\prime}$ contains the following two axioms in addition to axioms of SET:

$$
\begin{aligned}
& \neg(S=\{\}) \rightarrow \operatorname{choose}\left(S \cup S^{\prime}\right) \Rightarrow \operatorname{choose}(S) \\
& \neg(S=\{\}) \rightarrow \operatorname{choose}(\operatorname{apply} f(S)) \Longrightarrow f(\operatorname{choose}(S))
\end{aligned}
$$

Compared with $\mathrm{SET}, \mathrm{SET}^{\prime}$ has a reduced degree of nondeterminism and is more efficient in computation. The refinement predicate is stronger than the equality predicate, because from $S=S^{\prime}$ we cannot infer choose $(S)=$ choose $\left(S^{\prime}\right)$ (choose is referentially opaque), but from $S \Rightarrow S^{\prime}$ we can infer 
$\operatorname{choose}(S) \Rightarrow \operatorname{choose}\left(S^{\prime}\right)$, and hence choose $(S)=\operatorname{choose}\left(S^{\prime}\right)$. Notice that such a refinement is not semantically sound if nondeterministic operators are referentially transparent. As an example, suppose that choose is referentially transparent, and we have in SET a union operator $U_{-}$: set, set $\rightarrow$ set defined by

$$
\begin{aligned}
& \{\} \cup S^{\prime}=S^{\prime} \\
& (a \circ S) \cup S^{\prime}=a \circ\left(S \cup S^{\prime}\right)
\end{aligned}
$$

From $S \cup S^{\prime}=S^{\prime} \cup S$, we infer by the substitutivity axiom choose $\left(S \cup S^{\prime}\right)=$ choose $\left(S^{\prime} \cup S\right)$. Applying the first conditional refinement rule once on each side of the equation, we get $\neg(S=\{\}) \wedge \neg\left(S^{\prime}=\{\}\right) \rightarrow$ choose $(S)=$ choose $\left(S^{\prime}\right)$, which leads to the collapse of the carrier set $A_{\text {atom }}$ to a single element in any SET'-model, since from $\neg(a \circ\{\}=\{\})$ and $\neg(b \circ\{\}=\{\})$, we infer choose $(a \circ\{\})=\operatorname{choose}(b \circ\{\})$ and hence $a=b$.

\section{CONCLUSION}

The refinement of nondeterministic data types is a process which gradually removes nondeterminism by making design decisions that lead to an efficient implementation. However, with the specification of nondeterministic data types formalized in equational logic, many desirable refinements become impossible to prove correct. Nondeterminism often has to be removed entirely in one refinement step, rather than gradually through many refinement steps. This problem is caused by the requirement of referential transparency imposed on nondeterministic operators.

We proposed an alternative formalization of nondeterministic data types, which imposes the requirement of referential transparency only on deterministic operators. With this approach, we can easily show the correctness of many desirable refinements. Furthermore, a well-defined notion of refinement can be built into nondeterministic data types as a refinement operator, which is reflexive, transitive, and monotonic. Using such an operator, the gradual removal of nondeterminism can be formulated as conditional refinement rules, whose stepwise application leads to efficient implementation.

\section{ACKNOWLEDGMENT}

We thank Lee Blaine for our collaboration on the DTRE program transformation system, which has been a source of inspiration for the ideas presented here. The first author also thanks Peter Ladkin and José Meseguer for helpful discussions. Comments from the anonymous referees helped improve the presentation of the paper.

\section{REFERENCES}

Bidort, M., Kreowski, H.-J., Lescanne, P., Orfins, F., And SAnnella, D. 1991. Algebraic system specification and development. In Lecture Notes in Computer Science. Vol. 501. Springer-Verlag, New York.

BIRD, R., MEETENS, L., AND WILE, D. 1987. A common basis for algorithmic specification and development. Tech. Rep. CS-N8702, Center for Mathematics and Computer Science, Amsterdam, 89-100.

ACM Letters on Programming Languages and Systems, Vol. 2, Nos. 1-4, March-December 1993. 
Blaine, L., AND GoldBerg, A. 1991. D'TRE-A semi-automatic transformation system. In Constructing Programs from Specifications, B. Möller, Ed. North-Holland, Amsterdam, $165-204$.

Broy, M., Möller, B., AND Pepper, P. 1986. Algebraic implementations preserve program correctness. Sci. Comput. Program. 7, 35-53.

Goguen, J. A., Thatcher, J. W., AND Wagner, E. G. 1987. An initial algebra approach to the specification, correctness and implementation of abstract data types. In Current Trends in Programming Methodology, Vol. 4: Data Structuring, R. T. Yeh, Ed. Prentice-Hall, Englewood Cliffs, N.J., 80-149.

GutTAG, J. V., AND HoRNing, J. J. 1978. The algebraic specification of abstract data types. Acta Inf. 10, 27-52.

Hesselink, W. 1988. A mathematical approach to nondeterminism in data types. ACM Trans. Program. Lang. Syst. 10, 1 (Jan.), 87-117.

Meseguer, J. 1992. Conditional rewriting logic as a unified model of concurrency. Theor. Comput. Sci. 96, 1 (April), 73-155.

Nipkow, T. 1986. Non-deterministic data types: models and implementations. Acta Inf. 22 , 629-661.

SANNELlA, D., AND TARLECKI, A. 1988. Toward formal development of programs from algebraic specifications: implementations revisited. Acta Inf. 25, 3, 233-281.

SøndergaARd, H., AND SESTOFT, P. 1990. Referential transparency, definiteness and unfoldability. Acta Inf. 27, 6 (May), 505-517.

Turski, W., And Maibaum, T. 1987. The Specification of Computer Programs. Addison-Wesley, Reading, Mass.

Received June 1993; revised November 1993; accepted November 1993 